\title{
Tarifliche Öffnung in Deutschland: Ausmaß, Determinanten, Auswirkungen
}

\author{
Tobias Brändle · Wolf Dieter Heinbach • Michael \\ F. Maier
}

Angenommen: 14. Februar 2011 / Online publiziert: 3. März 2011

(C) Institut für Arbeitsmarkt- und Berufsforschung 2011

Zusammenfassung Im internationalen Vergleich behauptet sich der deutsche Arbeitsmarkt nach der durch die Finanzkrise ausgelösten Wirtschaftskrise relativ gut. Dies war lange Zeit nicht der Fall: Noch bis vor wenigen Jahren wurden die deutschen Arbeitsmarktinstitutionen immer wieder kritisiert, bei zunehmendem internationalen Wettbewerb zu wenige Möglichkeiten zur Anpassung von Lohnstruktur und Beschäftigung zu ermöglichen. Dabei zeigt sich bei genauerer Betrachtung, dass seit spätestens Mitte der 1990er Jahre ein Reformprozess in Gang gekommen ist, welcher das formal starre System der Lohnsetzung zunehmend flexibilisiert und dezentralisiert.

Dieser Beitrag fasst die Entwicklungen der in Tarifverträgen verankerten Flexibilisierungsmöglichkeiten zusammen. Diese haben in Form von Öffnungsklauseln stark zugenommen. Der Beitrag gibt zunächst einen Überblick über

Der Beitrag entstand im Rahmen des von der Deutschen Forschungsgemeinschaft (DFG) unterstützten Projekts „Auswirkungen von Tarifverträgen und Entgeltöffnungsklauseln auf die Lohnflexibilität und die betriebliche Entwicklung“ (DFG-Förderkennzeichen Ro 534/7-3). Die Autoren danken dem Herausgeber und einem anonymen Gutachter für wertvolle Hinweise und Kommentare.

T. Brändle

Eberhard Karls Universität Tübingen, Melanchthonstr. 30, 72074 Tübingen, Deutschland

e-mail: tobias.braendle@uni-tuebingen.de

W.D. Heinbach $(\bowtie)$

Ministerium für Wissenschaft, Forschung und Kunst Baden-Württemberg, Universität Hohenheim und IAW Tübingen, Königstraße 46, 70173 Stuttgart, Deutschland

e-mail: wolf.heinbach@mwk.bwl.de

M.F. Maier

ZEW Mannheim, L 7,1, 68161 Mannheim, Deutschland e-mail: maier@zew.de die bisherige Forschung im Bereich der Flexibilisierung von tariflichen Vereinbarungen und zeigt die bestehenden Lücken auf. Durch Generierung eines neuen Datensatzes (IAW-Öffnungsklauseldatensatz) ist es möglich, das Ausmaß der tariflichen Öffnung zu quantifizieren. Auf dieser Basis werden die Determinanten der Einführung von Öffnungsklauseln auf Branchenebene sowie deren betriebliche Anwendung auf Firmenebene ermittelt. Der letzte Teil des Beitrags widmet sich den wesentlichen Zusammenhängen zwischen tarifvertraglicher Öffnung und betrieblicher Entwicklung, mit besonderem Blick auf die Lohnstruktur und das Beschäftigungswachstum. Im Ergebnis zeigt sich, dass Tariföffnungsklauseln im Zeitablauf eine weite Verbreitung finden und dass mit ihnen eine Diversifizierung der Lohnstruktur sowie eine Steigerung des Beschäftigungswachstums verbunden ist.

Schlüsselwörter Tarifverträge · Öffnungsklauseln · Löhne · Beschäftigung

JEL Klassifikationen $\mathrm{J} 51 \cdot \mathrm{J} 63 \cdot \mathrm{C} 21$

\section{Flexibilisation in the German system of wage bargaining: extent, determinants, impacts}

\begin{abstract}
Given the recent financial crisis, the German labour market performs relatively well. This has not been the case until recent years: collective bargaining and the rigid system of wage setting have been often cited as one of the reasons for Germany's high structural unemployment. Contrary, a reform process has started at least since the mid1990s, introducing measures of flexibility and decentralization to the formally rigid system of wage setting.

This article summarises the developments of possible and actual flexibilisation of collective bargaining contracts via so
\end{abstract}


called hardship or opening clauses. A literature review sums up the scientific progress that had been made at the beginning of the research project and shows which gaps were to fill. Lacking data, the first parts of the project investigate on the actual existence and shape of opening clauses and create a new data set to measure the incidence of opening clauses in collective bargaining agreements in West German manufacturing sector for the years 1995 to 2007, the IAW data set on opening clauses. Using this, we analyse the determinants of the introduction of opening clauses on the industry level and of their actual implementation on the firm level. The latter parts of the project focus on the relation between opening clauses and measures of firm performance, especially considering wages and job growth. The results show that opening clauses are more and more common and that a more diversified wage structure and higher job growth is observed.

Keywords Collective bargaining - Opening clauses . Wages $\cdot$ Job flows

JEL Classification $\mathrm{J} 51 \cdot \mathrm{J} 63 \cdot \mathrm{C} 21$

\section{Einleitung}

In der aktuellen, durch die Finanzkrise ausgelösten Wirtschaftskrise entwickelt sich der deutsche Arbeitsmarkt im internationalen Vergleich relativ gut. Dies war bis vor wenigen Jahren nicht der Fall. Die damals noch höhere, strukturelle Arbeitslosigkeit führte dazu, dass der institutionelle Rahmen des deutschen Arbeitsmarkts grundlegend hinterfragt wurde. Vor diesem Hintergrund wurde argumentiert, dass das wesentlich von kollektiver Lohnsetzung geprägte Entgeltsystem keine hinreichende Flexibilität auf der Betriebsebene zuließe, diese jedoch im Zeitalter der Globalisierung mehr und mehr notwendig werde. Dabei ist in diesem Zusammenhang immer noch fraglich, ob den heterogenen Anforderungen an den Arbeitsmarkt aus einer zunehmenden Internationalisierung von Märkten und Unternehmen sowie eines immer schneller fortschreitenden technologischen Wandels überhaupt begegnet werden kann. Unabhängig davon sind sowohl das Ausmaß und die Nutzung bestehender tarifvertraglicher Flexibilisierungspotenziale als auch ihre Wirkungen bisher nur vereinzelt Gegenstand der Forschung (Fitzenberger und Franz 1999; Franz und Pfeiffer 2003). In jüngerer Zeit erfahren tarifvertragliche Flexibilisierungsmöglichkeiten jedoch stärkere Aufmerksamkeit (z.B. Gürtzgen 2008; Kohaut und Schnabel 2007; Kohn und Lembcke 2007).

Eine mögliche Flexibilisierung der tarifvertraglichen Bestimmungen - die im Fokus dieses Forschungsprojekts steht - ist die Einführung so genannter Tariföffnungsklauseln, mit deren Anwendung im Rahmen des Tarifvertrags vom Günstigkeitsprinzip nach §4 Abschn. 3 Tarifvertragsgesetz abgewichen werden kann. Durch die Öffnung der als zu starr angesehenen Flächentarifverträge soll erreicht werden, dass auf eine wirtschaftliche Krisensituation des Betriebs durch die flexible Gestaltung von Arbeitszeiten und - unter bestimmten Voraussetzungen - auch von Entgelten schnell und effektiv reagiert werden kann. Gleichzeitig werden die Vorteile einer einheitlichen, für alle tariflich gebundenen Beschäftigten verbindlichen Regelung der Arbeitsbedingungen und der Entlohnung beibehalten.

Bisherige Arbeiten zum Thema Öffnungsklauseln konzentrieren sich vor allem auf die Fragen, welche Öffnungsklauseln sich in welchen Bereichen etabliert haben (Bispinck 2003), oder ob sie in der betrieblichen Praxis angewendet werden (Franz und Pfeiffer 2003). Allerdings lagen längere Zeit kaum umfassende oder repräsentative Informationen über Verbreitung und Inanspruchnahme tarifvertraglicher Öffnungsklauseln und deren Wirkungen vor. Grundsätzliches Ziel ist es daher, sowohl die bestehenden Möglichkeiten als auch die tatsächliche Inanspruchnahme der tarifvertraglichen Flexibilität im deutschen Lohnbildungssystem aufzudecken und darauf aufbauend ihre Auswirkungen auf die Lohnstruktur und die Entwicklung betrieblicher Beschäftigung zu untersuchen. So ermitteln Burda et al. (2008) beispielsweise die Unterschiede zwischen tarifgebundenen und nicht-tarifgebundenen Arbeitnehmern entlang der Lohnverteilung. Gürtzgen (2008) analysiert die Effekte der Tarifbindung auf die Löhne unter Verwendung des LIAB-Datensatzes für den Zeitraum 1995-2002 und berücksichtigt dabei auch die Selektion von Arbeitnehmern in tarifgebundene Betriebe. In dieser Arbeit werden ergänzend auch die Informationen zum Ausmaß und zur Anwendung tarifvertraglicher Öffnungsklauseln verwendet.

Von zentraler Bedeutung ist die Frage, ob und in welchem Maße herkömmliche tarifvertragliche Rahmenbedingungen den Betrieben bereits eine ausreichend flexible Anpassung und Differenzierung der Entgelte ermöglichen. Deshalb soll analysiert werden, ob die zusätzliche Möglichkeit der Flexibilisierung durch Öffnungsklauseln von den Betrieben genutzt wird und ob diese zu einer günstigeren betrieblichen Entwicklung beiträgt. Insgesamt zielt das Forschungsprojekt somit auf eine Beantwortung der Frage, ob eine flexiblere Lohnanpassung und -differenzierung eine sinnvolle und wirksame Antwort auf die Anforderungen heterogener Arbeitsmärkte darstellen kann.

Der weitere Text gliedert sich wie folgt: In Abschn. 2 wird die Entwicklung der Tarifpolitik von den 1990er Jahren ausgehend vorgestellt. Die Beschreibung der damit einhergehenden Entwicklung der tarifvertraglichen Öffnung folgt in Abschn. 3. Welche Faktoren zu einer Tariföffnung führen, wird in Abschn. 4 ermittelt, bevor in Abschn. 5 der Zusammenhang zwischen der Existenz sowie der Anwendung von 
Öffnungsklauseln und der betrieblichen Entwicklung untersucht wird. Abschn. 6 fasst die Ergebnisse zusammen und schließt mit der Diskussion wirtschaftspolitischer Implikationen.

\section{Vier empirische Trends der Tarifpolitik}

Seit Beginn der 1990er Jahre lassen sich vier Entwicklungen erkennen, anhand derer die zunehmende Bewegung im bestehenden System tarifvertraglicher Verhandlungen in Deutschland deutlich wird. Als Erstes zeigt sich ein Rückgang des Anteils der Betriebe und gleichzeitig der Beschäftigten, für die ein Tarifvertrag Gültigkeit hat. Waren im Produzierenden Gewerbe im früheren Bundesgebiet im Jahr 1993 noch $84 \%$ der Betriebe und $95 \%$ der Beschäftigten tarifgebunden, so nahm dieser Anteil seitdem kontinuierlich ab. Im Jahr 2005 lag er nur noch bei $47 \%$ der Betriebe bzw. $64 \%$ der Beschäftigten (Kohaut und Schnabel 2003b; Heinbach 2009).

Als Zweites kann die gestiegene Zahl der insgesamt vereinbarten kollektiven Verbands- und Firmentarifverträge ausgemacht werden. Ein Erklärungsansatz hierfür ist, dass zusätzlich zu den bestehenden Flächentarifverträgen separate tarifvertragliche Regelungen auf der Betriebsebene vereinbart werden. Darüber hinaus zeigt sich ein Rückgang der kollektiven Tarifbindung auf Verbandsebene, der sich in einer steigenden Zahl der Haus- oder Firmentarifverträge widerspiegelt. Der Anteil an Hausoder Firmentarifverträgen verdreifachte sich zwischen 1990 und 2004 nahezu (Ellguth und Kohaut 2007; Heinbach 2009). Der Wechsel von einem Flächentarifvertrag zu einem Haus- oder Firmentarifvertrag stellt dabei eine Flexibilisierung dar, die Vorteile kollektivvertraglicher Regelungen erhält, gleichzeitig jedoch betriebsspezifische Anforderungen in den vertraglichen Bestimmungen stärker berücksichtigt.

Drittens zeigt sich, dass tarifvertragliche Regelungen zunehmend nicht generell für alle Beschäftigten gelten. Dies ist mit $\S 4$ Abschn. 1 des Tarifvertragsgesetzes vereinbar, der die Anwendung von Tarifverträgen nur für Gewerkschaftsmitglieder vorschreibt. Die Analyse entsprechender Merkmale der Verdienststrukturerhebung 2001 zeigt, dass ein Großteil der tarifgebundenen Betriebe nahezu alle Beschäftigten nach Tarifvertrag entlohnt, es aber eine ganze Reihe von Betrieben gibt, bei denen nur ein Teil der Beschäftigten der Tarifbindung unterliegt (Fitzenberger et al. 2008; Heinbach 2009).

Diese Entwicklungen zeugen von einem sich erhöhenden Druck auf die tarifpolitischen Vertragsparteien. Mit Blick auf die zunehmende Flucht aus kollektiven Tarifverträgen haben Arbeitgeber- und Arbeitnehmervertreter viertens Tarifverträge auf dem Verhandlungsweg flexibilisiert.
Damit verbunden sind so genannte betriebliche Bündnisse für Arbeit oder Standortpakte (Hübler 2005), die Einstellung von Zeit- und/oder Leiharbeitern, für welche andere tarifliche Regelungen gelten (Antoni und Jahn 2009), oder die Einführung von Öffnungsklauseln, die unter bestimmten Voraussetzungen Abweichungen von tarifvertraglichen Regelungen zulassen (Kohaut und Schnabel 2007; Heinbach 2007). Im Rahmen tarifvertraglicher Öffnungsklauseln können beispielsweise Entgelte abgesenkt oder Arbeitszeiten ohne entsprechenden Lohnausgleich verlängert oder verkürzt werden. Die Flexibilisierung tarifvertraglicher Bestimmungen mit Hilfe von Öffnungsklauseln steht im Fokus der folgenden Untersuchung.

\section{Ausmaß der tarifvertraglichen Öffnung}

Die hier betrachtete tarifliche Öffnung beschreibt die Möglichkeit, innerhalb eines Tarifvertrags Entgelte oder Arbeitsbedingungen von Beschäftigten unter gewissen Umständen auf betriebsspezifische Bedürfnisse anzupassen. Bislang bestehen keine umfassenden Untersuchungen über die generelle Verbreitung tarifvertraglicher Öffnung. Die Verbreitung tarifvertraglicher Öffnungsklauseln wird dabei zunächst als potenziell anwendbare, im Tarifvertrag verankerte Regelung betrachtet (Ausmaß der tarifvertraglichen Öffnung). Davon unterscheidet sich die tatsächliche Inanspruchnahme bzw. Anwendung der Öffnungsklauseln auf betrieblicher Ebene. Heinbach (2005) führt daher eine Vollerhebung in allen 93 Tarifbereichen ${ }^{1}$ des Produzierenden Gewerbes im Land Baden-Württemberg durch (IAW-Öffnungsklauseldatensatz). ${ }^{2}$ In einer Textauswertung wurden alle schriftlichen Regelungen in den betreffenden Flächentarifverträgen aus den Veröffentlichungen des WSI-Tarifarchivs und des Tarifregisters beim Ministerium für Arbeit und Soziales Baden-Württemberg ausgewertet. Im resultierenden IAW-Öffnungsklauseldatensatz mit insgesamt rund 240 Variablen ergibt sich ein exaktes Bild der in den betreffenden Tarifverträgen verankerten Öffnungs- und Härtefallklauseln. Neben detaillierten Schilderungen der genauen Ausgestaltung der entsprechenden Öffnungsklauseln sind auch der Einführungszeitpunkt und gegebenenfalls die Laufzeit erfasst. Daneben stehen Angaben zur Form der Beteiligung der Betriebsräte bzw. der

\footnotetext{
${ }^{1}$ Ein Tarifbereich ist als Geltungsbereich eines Tarifvertrages über drei Aspekte definiert: Erstens fachlich - ein Tarifvertrag wird in einer bestimmten Branche abgeschlossen (beispielsweise in der Metallindustrie). Zweitens regional - ein Tarifvertrag gilt immer für eine bestimmte Tarifregion (beispielsweise Nordwürttemberg/Nordbaden). Drittens persönlich - ein Tarifvertrag gilt, wenn beide Tarifparteien, also Arbeitgeber und Arbeitnehmer, Mitglied eines tarifschließenden Verbandes sind.

${ }^{2}$ Für die weiteren Analysen wurde der IAW-Öffnungsklauseldatensatz bis zum Jahr 2007 und für die Tarifbereiche im Produzierenden Gewerbe in Westdeutschland erweitert.
} 


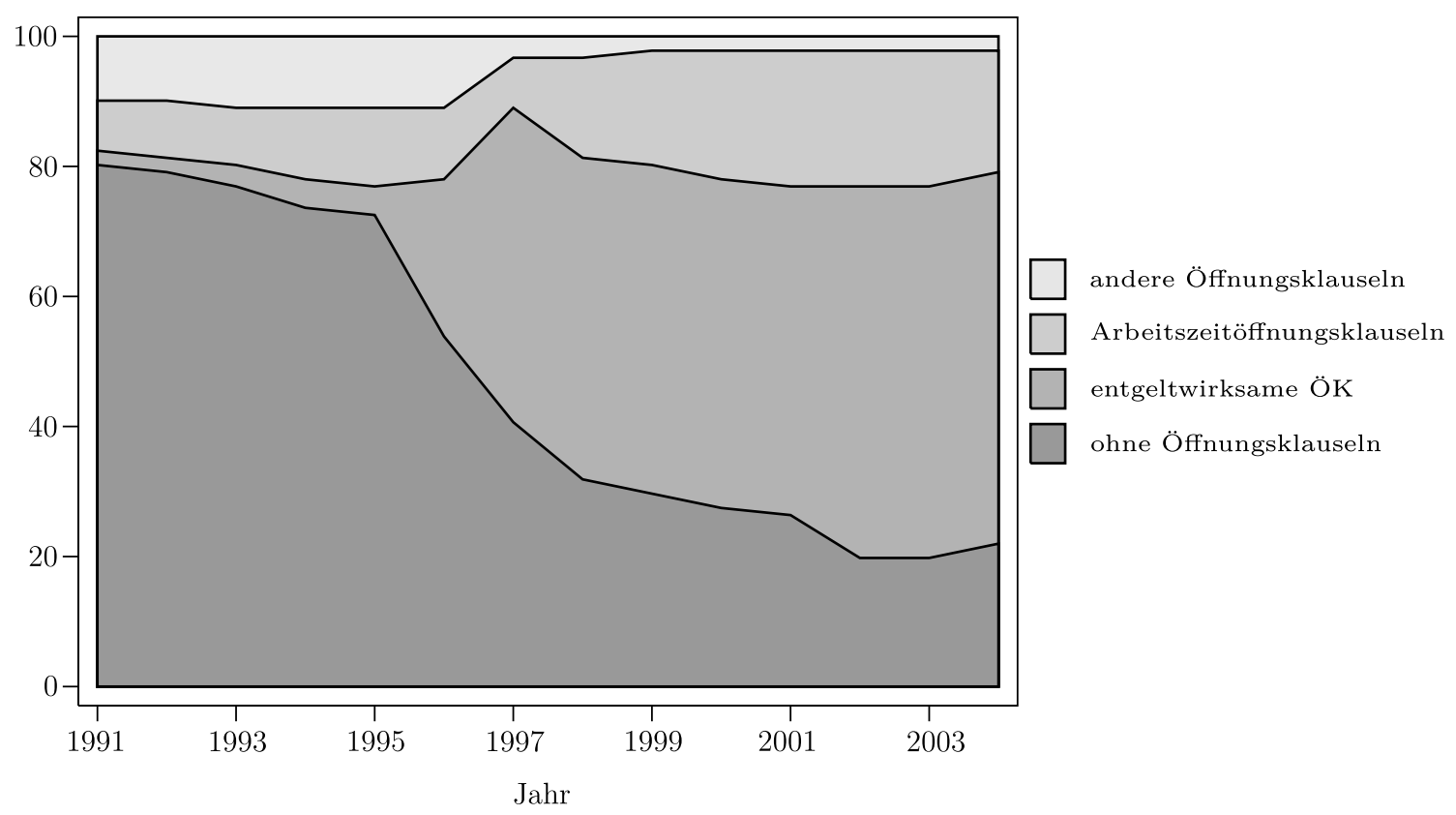

Abb. 1 Entwicklung der Tarifbereiche mit Öffnungsklauseln im Zeitraum von 1991 bis 2004, Produzierendes Gewerbe in Baden-Württemberg, Angaben in Prozent. Quelle: in Anlehnung an Heinbach (2007)

Tarifvertragsparteien und zu den Voraussetzungen für einen betrieblichen Einsatz von Öffnungsklauseln zur Verfügung.

Aus den Daten lassen sich für die einzelnen Tarifbereiche verschiedene Typen von Öffnungsklauseln unterscheiden. Jeder Tarifbereich gehört dabei eindeutig einem Öffnungsklauseltyp an. Tarifverträge mit allgemeinen Klauseln oder Klauseln, die zum Beispiel nur den Auszahlungszeitpunkt für Jahressonderzahlungen oder Urlaubsgeld betreffen, werden zu ,,andere Öffnungsklauseln“, Tarifverträge ohne jegliche Flexibilisierungsmöglichkeit zu „ohne Öffnungsklauseln“ zusammengefasst. Sieht ein Tarifvertrag mehrere Öffnungsklauseln zum Beispiel zu Entgelt, Arbeitszeit und Kleinbetrieben vor, wird dieser der Gruppe „Öffnungsklauseln mit Entgeltwirkung“ zugeordnet. Existieren nur andere Öffnungsklauseln und Arbeitszeitöffnungsklauseln, die nicht entgeltwirksam sind, so wird der Tarifvertrag der Gruppe „Arbeitszeitöffnungsklauseln“ zugeordnet. Annahmegemäß kann ein Tarifbereich nicht zwei Typen gleichzeitig angehören.

Aus der Verdienststrukturerhebung werden Beschäftigtenanteile für die jeweiligen Tarifbereiche ermittelt und über den Tarifvertragsschlüssel mit dem IAW-Öffnungsklauseldatensatz verknüpft. Aus dem Anteil der Beschäftigten, die einem Tarifvertrag mit einer bestimmten Öffnungsklausel unterliegen, ergibt sich ein Maß für die Verbreitung tarifvertraglicher Öffnungsklauseln. Innerhalb der Tarifvertragslandschaft bestehen einzelne Tarifverträge, die für eine Vielzahl von Betrieben und Beschäftigte gelten und damit eine dominante Position einnehmen. Daraus folgt, dass diese einzelnen Tarifverträge auch großen Einfluss auf das Ausmaß der tarifvertraglichen Öffnung haben. Sahen im Jahr 1991 nur rund $2 \%$ der Tarifbereiche entgeltwirksame Öffnungsklauseln und $7 \%$ der Tarifbereiche Arbeitszeitöffnungsklauseln vor, so existieren seit der Einführung in der Metallund Elektroindustrie 1997 bzw. 2001 in über der Hälfte der Tarifbereiche solche Öffnungsklauseln, die $81 \%$ der Beschäftigten umfassen (Abb. 1, Heinbach 2006). Bezüglich der tatsächlichen Anwendung findet Heinbach (2005), dass ein Großteil der Öffnungsklauseln, die die Arbeitszeit betreffen, durch freiwillige Betriebsvereinbarungen umgesetzt werden, während bei entgeltrelevanten Öffnungsklauseln in verstärktem Maße die Tarifvertragsparteien eingebunden sind.

Die gestiegene Heterogenität tarifvertraglicher Regelungen und insbesondere die inzwischen sehr unterschiedliche Ausgestaltung der Flexibilisierung durch Öffnungsklauseln sind bis jetzt noch wenig beachtet. Insbesondere branchen- und regionalspezifische Gründe führen dazu, dass sich Öffnungs- und Härtefallklauseln in ihrer formalen und inhaltlichen Ausgestaltung zum Teil erheblich unterscheiden. Daneben spielt das Umfeld der Einführung, also die Wettbewerbssituation und der Zeitpunkt der Verhandlungen, eine entscheidende Rolle. Heinbach und Schröpfer (2007) verwenden daher den IAW-Öffnungsklauseldatensatz, um die Heterogenität von Tarifvereinbarungen in Bezug auf mögliche Flexibilisierungsregelungen mit Hilfe von multivariaten Analysemethoden herauszuarbeiten.

Auf Basis des IAW-Öffnungsklauseldatensatzes lassen sich 37 verschiedene Kombinationen von Öffnungsmöglichkeiten ermitteln, die von Heinbach und Schröpfer (2007) 
Tab. 1 Bedeutung der Tarifbereichstypen gemessen am Anteil tarifgebundener Beschäftigter

Quelle:

Verdienststrukturerhebung 1995/2001, IAW-

Öffnungsklauseldatensatz, eigene Darstellung, Heinbach und Schröpfer (2007)

\begin{tabular}{|c|c|c|c|c|}
\hline \multirow[t]{2}{*}{ Typ } & \multicolumn{2}{|l|}{1995} & \multicolumn{2}{|l|}{2001} \\
\hline & $\begin{array}{l}\text { Beschäftigten- } \\
\text { anteil }\end{array}$ & $\begin{array}{l}\text { Zahl der } \\
\text { Tarifbereiche }\end{array}$ & $\begin{array}{l}\text { Beschäftigten- } \\
\text { anteil }\end{array}$ & $\begin{array}{l}\text { Zahl der } \\
\text { Tarifbereiche }\end{array}$ \\
\hline Jahressonderzahlung verschieben & $53,6 \%$ & 3 & $0,1 \%$ & 1 \\
\hline Öffnungsklausel-Kombinationen & $9,3 \%$ & 8 & $82,6 \%$ & 29 \\
\hline Arbeitszeitkorridor & & & $6,1 \%$ & 4 \\
\hline Urlaubsgeld verschieben & $0,1 \%$ & 2 & $0,3 \%$ & 1 \\
\hline Arbeitszeit absenken & $2,6 \%$ & 2 & $1,8 \%$ & 9 \\
\hline Grundentgelt absenken & & & $0,2 \%$ & 2 \\
\hline keine Öffnungsklausel & $34,5 \%$ & 45 & $8,9 \%$ & 14 \\
\hline
\end{tabular}

durch eine systematische und differenzierte Typisierung mittels Clusteranalyse, der eine Korrespondenzanalyse vorangestellt ist, strukturiert werden. Insgesamt unterscheiden Heinbach und Schröpfer (2007) 7 Cluster, wobei eines keine Öffnungsklauseln beinhaltet und vier jeweils eine bestimmte Öffnungsklausel. Ein weiteres Cluster besteht aus Tarifbereichen, die eine Kombination mehrerer Öffnungsklauseln darstellen. ${ }^{3}$

Werden die Beschäftigtenanteile aus der Verdienststrukturerhebung Baden-Württemberg den entsprechenden Tarifbereichen zugespielt, so zeigt sich, dass sich der Anteil der tarifgebundenen Beschäftigten ohne Flexibilisierungsmöglichkeit von mehr als $34 \%$ in 45 Tarifbereichen im Jahr 1995 auf lediglich noch $9 \%$ in 14 Tarifbereichen im Jahr 2001 erheblich verringert. Die Mehrheit der Beschäftigten, die 1995 von höchstens einer Öffnungsklausel betroffen waren, unterliegt im Jahr 2001 tarifvertraglichen Regelungen mit mehreren Öffnungsklauseln (Tab. 1).

Vor dem Hintergrund der deutlichen Unterschiede der Öffnungsmöglichkeiten in den verschiedenen Tarifbereichen werden die Öffnungsklausel-Typen verwendet, um Branchentarifverträge in Bezug auf ihre tarifliche Öffnung zu charakterisieren. Ist der Typ-spezifische Anteil der tarifgebundenen Beschäftigten in einer Branche ausreichend hoch $(>75 \%)$, so wird im Folgenden von einem branchenspezifischen Tarifvertragstyp gesprochen. Dies ist für 24 Branchen im Jahr 1995 und 22 Branchen im Jahr 2001 der Fall. Eine Einteilung in die Typen „keine Öffnungsklauseln“, „Arbeitszeit-Öffnungsklauseln“ und „entgeltwirksame Öffnungsklauseln" ermöglicht es, verschiedene Datensätze wie das IAB-Betriebspanel oder den Linked-Employer-Employee-Datensatz des IAB (LIAB) um die Informationen aus dem IAW-Öffnungsklauseldatensatz zu ergänzen, was in den im Folgenden beschriebenen Analysen genutzt werden kann.

Gerade im Bereich entgeltwirksamer Flexibilisierung kann eine stark zunehmende Verbreitung im Zeitraum von

${ }^{3}$ Eine Zuordnung der 7 Cluster von Heinbach und Schröpfer (2007) zu den Öffnungsklauseltypen nimmt Heinbach (2009, S. 51) vor.
1991 bis 2004 beobachtet werden. Die in der Diskussion um den Flächentarifvertrag immer wieder auftauchenden Forderungen nach dessen stärkerer Dezentralisierung stehen dazu im Kontrast, denn für die große Mehrheit der tariflich gebundenen Beschäftigten sind Abweichungen vom Tarifvertrag bereits verhandelbar.

Empirische Ergebnisse auf Basis des IAB-Betriebspanels zeigen allerdings, dass eine Vielzahl der Betriebe über die Existenz von Tariföffnungsklauseln schlecht informiert ist (Kohaut und Schnabel 2007; Heinbach und Schröpfer 2008). Auch Franz und Pfeiffer (2006) bestätigen diese „Unkenntnis“ auf Basis einer eigenen Unternehmensbefragung. Deshalb ergänzen Heinbach und Schröpfer (2008) die Angaben des IAB-Betriebspanels um zusätzliche Informationen aus dem IAW-Öffnungsklauseldatensatz. Für alle Betriebe, die im IAB-Betriebspanel angeben, nicht über Tariföffnungsklauseln informiert zu sein, wird durch Informationen über Branche und Region eines Betriebs die Zugehörigkeit zu einem bestimmten Tarifbereich ermittelt. Ist dies möglich, werden dementsprechend die Angaben zur Existenz von Tariföffnungsklauseln aus dem IAW-Öffnungsklauseldatensatz übernommen. Das so erweiterte IAB-Betriebspanel ermöglicht detaillierte Untersuchungen der Wirkung von Flexibilisierungspotenzialen und der betrieblichen Anwendung von tarifvertraglichen Öffnungsklauseln.

\section{Determinanten der Tariföffnung}

\subsection{Faktoren der Einführung von Öffnungsklauseln}

Die zunehmende Verbreitung tarifvertraglicher Öffnungsklauseln, die zeitlich und inhaltlich unterschiedliche Entwicklungspfade vollziehen (Heinbach und Schröpfer 2007; Heinbach 2009), führt zu der Frage, ob die Einführung tarifvertraglicher Öffnungsklauseln in den 1990er Jahren durch bestimmte ökonomische Rahmenbedingungen begünstigt bzw. verschärft wurde. Unter Berücksichtigung des Einführungszeitpunktes tarifvertraglicher Öffnungsklauseln im IAW-Öffnungsklauseldatensatz und detaillierter Daten zu 
Struktur und Lage der Branchen des Produzierenden Gewerbes in Westdeutschland im Zeitraum 1993 bis 2002 wird der Einfluss verschiedener Größen auf die Einführung von Öffnungsklauseln untersucht (Spindler 2007). Basis für die Branchenmerkmale bilden die OECD-STAN-Database (OECD 2008) und das IAB-Betriebspanel (Kölling 2000). Während die OECD-Daten in erster Linie Aufschluss über wichtige makroökonomische Faktoren wie z.B. Output, Kapitalstock, internationaler Handel sowie Arbeitskosten auf Branchenebene geben, lassen sich aus dem IAB-Betriebspanel hochgerechnete Informationen über die jeweilige Branchenstruktur gewinnen, wie beispielsweise die Betriebsgrößenstruktur oder den Anteil tarifgebundener Betriebe. Des Weiteren werden die Insolvenzstatistik des Statistischen Bundesamts sowie Geschäftsklimaindikatoren des ifo Instituts (Abberger et al. 2007) als Indikatoren für die gesamtwirtschaftliche Lage genutzt. Die Informationen über die Einführung von Öffnungsklauseln werden dem IAW-Öffnungsklauseldatensatz entnommen (Heinbach 2005), wobei Schwerpunkttarifverträge auf Branchenebene gebildet werden (Heinbach 2009; Heinbach und Schröpfer 2008). ${ }^{4}$ Spindler (2007) bestimmt die Determinanten mit Hilfe verschiedener binärer (Paneldaten-)Modelle.

Im Ergebnis zeigt sich, dass vor allem steigende Arbeitskosten, ein zunehmender Anteil an mittelgroßen Betrieben und ein abnehmender Anteil an tarifgebundenen Betrieben innerhalb einer Branche sowie ein Anstieg der Exporttätigkeit auf Branchenebene jeweils in positivem Zusammenhang mit der Flexibilisierung eines Schwerpunkttarifvertrags stehen. Faktoren wie das Ausmaß der Produktion gemessen als Wert aller innerhalb eines Jahres produzierten Waren und Güter in einer Branche und deren Veränderung, der Anteil tarifvertraglich Beschäftigter, die Anzahl an Unternehmensinsolvenzen innerhalb einer Branche oder die Ertragslage weisen dagegen keine statistische Signifikanz auf.

Es zeigt sich also, dass die Einführung tarifvertraglicher Öffnungsklauseln im Zusammenhang mit der jeweiligen ökonomischen Situation der Branche steht. Tarifvertragliche Öffnungsklauseln sind damit eine sinnvolle Reaktion auf ein vom zunehmenden internationalen Wettbewerb geprägtes ökonomisches Umfeld und spiegeln die steigenden betrieblichen Flexibilisierungserfordernisse hinsichtlich der Gestaltung von Entgelt und Arbeitszeit wider.

\subsection{Exporttätigkeit und Öffnungsklauseln}

Da aus Sicht der Arbeitgeber vor allem der gestiegene internationale Wettbewerb eine verstärkte Flexibilisierung von

\footnotetext{
${ }^{4}$ Die Schwerpunkttarifverträge werden auf Basis der Verknüpfung von IAW-Öffnungsklauseldatensatz und Verdienststrukturerhebung bestimmt. Unter Annahme einer konstanten Struktur der tarifgebundenen Beschäftigten in Bezug auf den für die Beschäftigten angewendeten Tarifvertrag (Referenzjahr 2001) wird der für eine Branche typische Tarifvertrag bestimmt, (Heinbach 2009, S. 55 ff.).
}

Tarifverträgen nötig macht, stellt sich die Frage, ob die betriebliche Anwendung tarifvertraglicher Öffnungsklauseln von Produktivitätsunterschieden der Firmen untereinander abhängt. Basierend auf mikroökonomischen Handelstheorien argumentieren zum Beispiel Bernard et al. (2003), dass internationaler Wettbewerb zu größerer Heterogenität zwischen Betrieben innerhalb der Branche führt. Exportorientierte Betriebe sind aufgrund ihrer hohen Produktivität in der Lage, ausländische Märkte zu erschließen. Je höher ihre Produktivität desto eher wird der Betrieb auch in stark entfernte Regionen exportieren, in denen er trotz hinzukommender Transportkosten noch günstigster Anbieter ist. Hingegen produzieren weniger produktive Betriebe lediglich für den inländischen Markt, stehen dabei aber unter dem Druck ausländischer (Import-)Konkurrenz. Steigt nun der internationale Wettbewerb, beispielsweise durch sinkende Transportkosten, so profitieren exportorientierte Unternehmen, während sich nicht-exportorientierte Unternehmen durch vermehrten Import von Waren ausländischer Unternehmen einer stärkeren Konkurrenz ausgesetzt sehen. Dies führt zu einer divergierenden Arbeitsnachfrage und damit verbundenen heterogenen Lohnsetzungstendenzen zwischen den Unternehmen einer Branche. Hieraus können Forderungen nach einer flexibleren Lohnsetzung innerhalb einer Branche folgen.

Heinbach und Schröpfer (2008) nutzen das um Informationen aus dem IAW-Öffnungsklauseldatensatz ergänzte IAB-Betriebspanel und insbesondere Angaben über die betriebliche Exportaktivität, den Exportanteil am Umsatz sowie Entfernungen zu Absatzmärkten, um die Hypothesen von Bernard et al. (2003) zu testen. Mit Hilfe eines LogitModells wird der Einfluss betrieblicher und branchenspezifischer Faktoren auf die Wahrscheinlichkeit der betrieblichen Anwendung von Tariföffnungsklauseln untersucht. Es zeigt sich, dass Betriebe, die nur in das EU-Ausland exportieren, seltener Öffnungsklauseln anwenden als nichtexportorientierte Betriebe. Dagegen finden sich keine Unterschiede zwischen allen anderen exportorientierten Betrieben und den nicht-exportorientierten Betrieben.

Somit kann empirisch nicht eindeutig bestimmt werden, ob bei wachsendem Wettbewerb weniger produktive Betriebe im Mittel häufiger Öffnungsklauseln anwenden. Allerdings sind die Ergebnisse mit der Hypothese einer Zunahme der Heterogenität der Arbeitsnachfrage kompatibel, da exportorientierte und damit produktivere Unternehmen weniger häufig Tariföffnungsklauseln nutzen.

\section{5 Öffnungsklauseln und betriebliche Entwicklung}

\subsection{Tarifvertragliche Lohnstruktur}

Auf Basis der Verknüpfung des IAW-Öffnungsklauseldatensatzes mit der Verdienststrukturerhebung der Jahre 1995 
und 2001, einem Linked-Employer-Employee Datensatz der Statistischen Ämter der Länder, untersucht Heinbach (2007) den Einfluss tariflicher Öffnungsklauseln auf die Lohnstruktur im Verarbeitenden Gewerbe. Bislang unterscheidet die empirische Literatur über gewerkschaftliche Lohnbildung in Deutschland meist zwei Arten kollektiver Lohnverhandlungen, nämlich Flächentarifverträge und Haus- oder Firmentarifverträge (Gerlach und Stephan 2006). Heinbach (2007) berücksichtigt zusätzlich Flächentarifverträge mit Arbeitszeitöffnungsklauseln oder mit entgeltrelevanten Öffnungsklauseln im Vergleich zu Flächentarifverträgen ohne Öffnungsklauseln..$^{5}$ Aus theoretischer Sicht ist der Effekt von Öffnungsklauseln auf die Lohnhöhe und auf die Lohnverteilung unbestimmt, je nachdem, wie hoch Lohnabschlüsse bei Vorliegen von Öffnungsklauseln ausfallen und wie viele Firmen dann tatsächlich von den vereinbarten Öffnungsklauseln Gebrauch machen (Fitzenberger und Franz 1999).

Im Ergebnis zeigen sich kollektiv verhandelte Löhne als höher und komprimierter im Vergleich zu individuell mit jedem einzelnen Beschäftigten verhandelte Löhne. Betriebe, in deren Tarifverträgen zusätzlich Öffnungsklauseln existieren, weisen eine stärker differenzierte Lohnstruktur auf. Während in Betrieben mit Arbeitszeitöffnungsklauseln ein eher höherer Lohn festzustellen ist und die Lohnspreizung geringer ausfällt, sind in Betrieben mit entgeltwirksamen Öffnungsklauseln sinkende Löhne zu verzeichnen. Bei ersteren existiert also eine Art Preis für Flexibilisierung. Im Falle von entgeltrelevanten Öffnungsklauseln ist dagegen zu vermuten, dass dieser Lohnaufschlag durch einen hohen Anteil an Firmen, die Öffnungsklauseln tatsächlich nutzen und damit den letztlich beobachteten Lohn senken, (über-)kompensiert wird. Es kann in diesem Zusammenhang zudem von einer negativen Selektion von Betrieben ausgegangen werden, die aufgrund anderer Faktoren a priori geringere Löhne zahlen und unter Umständen dann noch zu Lohnsenkungen aufgrund von Öffnungsklauseln greifen (müssen). ${ }^{6}$ Ebenso ist der Anteil der Firmen, die weiterhin über Tarif zahlen, relativ hoch (Kohaut und Schnabel 2003a). Dies zeigt aber, dass bei der Beurteilung der Ergebnisse zwischen der Existenz einer Öffnungsklausel im ausgehandelten Tarifvertrag und der tatsächlichen Anwendung unterschieden werden muss. Es ist also festzuhalten, dass durch Öffnungsklauseln die Tariflandschaft weiter differenziert und dezentralisiert wird und Firmen mehr und mehr ihre Lohnsetzungspräferenzen über die Wahl eines für sie geeigneten Tarifregimes wählen können.

\footnotetext{
${ }^{5}$ Der Typ ,,andere Öffnungsklauseln“ wird aufgrund der geringen Relevanz für die Untersuchung der Lohnstruktur dem Regime „ohne Öffnungsklauseln“ zugeordnet. Tarifverträge mit sowohl Arbeitszeit- als auch Entgeltöffnungsklauseln werden letzteren zugeordnet.

${ }^{6} \mathrm{Da}$ es im betrachteten Zeitraum jedoch zu einer vermehrten Einführung von Öffnungsklauseln gekommen ist, können diese Effekte nicht als kausal interpretiert werden.
}

Die Verknüpfung von IAW-Öffnungsklauseldatensatz und IAB-Betriebspanel (Heinbach und Schröpfer 2008) ermöglicht es, die Auswirkungen tarifvertraglicher Öffnungsklauseln auf die Lohnstruktur auf Basis des LIAB zu untersuchen. Sowohl Informationen zur Existenz von Tariföffnungsklauseln aus dem IAW-Öffnungsklauseldatensatz als auch über deren Anwendung aus den Informationen des IAB-Betriebspanels können darin genutzt werden. In den Jahren 2005 und 2007 weisen $72 \%$ bzw. $78 \%$ aller tarifgebundenen Betriebe mindestens eine Öffnungsklausel in ihrem Tarifvertrag auf. Im Hinblick auf die Beschäftigten liegt dieser Anteil mit knapp $90 \%$ sogar noch höher. Anwendung finden tarifvertragliche Öffnungsklauseln in $13 \%$ der Betriebe mit Flächentarifvertragsbindung im Jahr 2005 (11\% in 2007), der Anteil der betroffenen Beschäftigten liegt bei $31 \%$ (33\% in 2007).

Mit Hilfe von Quantilsregressionen ermittelt Maier (2009), dass bei Existenz tarifvertraglicher Öffnungsklauseln tarifgebundene Beschäftigte durchschnittlich einen höheren Lohn als Beschäftigte mit individuellen Lohnverhandlungen aufweisen. Dies gilt vor allem im unteren Bereich der Lohnverteilung, während im oberen Bereich ein Lohnabschlag im Vergleich zu Beschäftigten in Tarifverträgen ohne Öffnungsklauseln zu beobachten ist. Somit geht die tarifliche Öffnung mit einer Kompression der Lohnverteilung einher. Dieses Bild zeigt sich auch, wenn neben der Existenz zusätzlich die Anwendung tarifvertraglicher Öffnungsklauseln berücksichtigt wird. Allerdings ist zu vermuten, dass auch diese Ergebnisse zu einem gewissen Grad durch die oben beschriebenen Selektionseffekte beeinflusst sind.

\subsection{Beschäftigungswachstum in Betrieben}

Neben dem Effekt tarifvertraglicher Öffnungsklauseln für die betriebliche Lohnstruktur stellt sich auch die Frage, ob Tariföffnungsklauseln einen Einfluss auf das betriebliche Beschäftigungswachstum haben. Für Betriebe des Verarbeitenden Gewerbes in Westdeutschland untersuchen Brändle und Heinbach (2010), ob mit tariflichen Öffnungsklauseln primär neue Arbeitsplätze geschaffen oder bestehende Arbeitsplätze gesichert werden. Aus theoretischer Sicht sind zwei Effekte denkbar. Erstens können Betriebe, welche Öffnungsklauseln anwenden, die Lohnkosten in Krisenzeiten senken und damit Arbeitsplätze erhalten. Zweitens könnte das Wissen um die Existenz von Öffnungsklauseln im Tarifvertrag dazu führen, dass Betriebe mehr Arbeitsplätze schaffen, welche dann später durch Anwendung derselben in ihrem Bestand gesichert werden können. Insgesamt kann daher eine positive Auswirkung auf das Beschäftigungswachstum erwartet werden. Der Einfluss auf den Arbeitsplatzumschlag, also die Summe von geschaffenen und abgebauten Arbeitsplätzen, ist zunächst unklar, da die beiden erwarteten Effekte in unterschiedliche Richtungen wirken. 
Auf Basis der Verknüpfung von IAB-Betriebspanel mit dem IAW-Öffnungsklauseldatensatz soll das PropensityScore-Matching-Verfahren die zu erwartende Selektion von Betrieben in verschiedene Tarifregime anhand beobachtbarer Variablen kontrollieren. Vergleiche mit gewöhnlichen Kleinstquadrate-Schätzungen zeigen, dass diese Art von Selektion nicht zu vernachlässigen ist. Bezüglich der Wirkung tarifvertraglicher Öffnung zeigt sich, dass Betriebe mit Öffnungsklauseln in deren Tarifverträgen eine signifikant niedrigere Rate des Arbeitsplatzabbaus aufweisen. Für die tatsächliche Inanspruchnahme von Tariföffnungsklauseln gilt dies jedoch nicht. Dies könnte darauf hinweisen, dass Tariföffnungsklauseln ausgehend von einem Modell der dynamischen Arbeitsnachfrage ex ante das Risiko für Anpassungskosten der Faktorinputs mindern und deshalb unabhängig von der Inanspruchnahme die betriebliche Beschäftigung steigen würde. Dem könnte entgegenwirken, dass Betriebe in einer schlechten wirtschaftlichen Situation dazu gezwungen sind, Arbeitsplätze abzubauen, obwohl sie Tariföffnungsklauseln anwenden (unbeobachtete negative Selektion). Die gegenläufigen Effekte könnten dazu führen, dass sich für diese Gruppe kein Unterschied ergibt. Andererseits finden Brändle und Heinbach (2010) keine Effekte von Tariföffnungsklauseln im Hinblick auf die Rate der Arbeitsplatzschaffung. Dies gilt sowohl für die Existenz als auch für die tatsächliche Inanspruchnahme tarifvertraglicher Öffnungsklauseln. Dies deutet darauf hin, dass Betriebe ihr Beschäftigungsniveau aufgrund der potenziell niedrigeren Arbeitskosten nicht über das Ausgangsniveau anheben, um dann vorhandene Flexibilisierungspotenziale zu Lasten der Beschäftigten zu nutzen. Insgesamt wachsen Betriebe mit tarifvertraglichen Öffnungsklauseln jährlich im Durchschnitt um 0,73 Prozentpunkte mehr als solche ohne. Man kann daraus jedoch nicht folgern, dass Betriebe mit Tariföffnungsklauseln grundsätzlich mehr Arbeitsplätze schaffen, sondern dass sie bestehende eher sichern. Tariföffnungsklauseln würden sich daher eher als Krisen- denn als generelles Beschäftigungsinstrument verstehen. Brändle und Heinbach (2010) bestätigen die Hypothese, dass beschäftigungspolitisch bereits wirksame institutionelle Maßnahmen im deutschen Tarifvertragssystem existieren. Tarifvertragliche Öffnung kann durch Flexibilisierung und Dezentralisierung zu mehr Beschäftigung in Betrieben führen, ohne die Vorteile kollektiver Lohnsetzung zu verlieren.

\section{Fazit}

Das deutsche Tarifvertragssystem unterliegt seit den 1990er Jahren einem Prozess hin zu einer flexibleren Gestaltung der kollektiven Lohnsetzung. Dies kann als eine Antwort auf die geänderten Anforderungen an das Lohnsetzungssystem angesehen werden, welche eine zunehmende Globalisierung und internationaler Wettbewerb mit sich bringen. Dabei sehen sich Betriebe mit immer kurzfristigeren Veränderungen auf einem bereits stark heterogenen Arbeitsmarkt konfrontiert. Ziel des Forschungsprojekts ist es darum, bestehende Möglichkeiten der flexiblen Entgeltanpassung im deutschen Lohnbildungssystem und deren tatsächliche Inanspruchnahme zu identifizieren sowie die damit verbundenen Auswirkungen auf die betriebliche Entwicklung, insbesondere die Lohnstruktur und das Beschäftigungswachstum zu untersuchen.

Grundlegend kann ein Trend hin zu einer flexibleren vertraglichen Gestaltung von Tarifverträgen beobachtet werden. Hierbei spielen verschiedene Faktoren eine Rolle. Im vorliegenden Beitrag liegt das Hauptaugenmerk auf tarifvertraglichen Öffnungsklauseln, deren Bedeutung sich in den Jahren 1991 bis 2001 beträchtlich steigerte - von etwa $20 \%$ der Beschäftigten, die einem Tarifvertrag mit enthaltener Öffnungsklausel unterstehen, zu einem Anteil von $81 \%$ (Heinbach 2005).

Eine detaillierte Textanalyse der einzelnen Tarifverträge zeigt eine beträchtliche Heterogenität tariflicher Öffnungsklauseln, aufgrund derer Heinbach und Schröpfer (2007) eine Typisierung vornehmen. Bestehende Öffnungsklauseln sind meist entgelt- oder arbeitszeitrelevant; die meisten stellen eine Kombination aus beidem dar. Aus den verschiedenen Typen von Tariföffnungsklauseln werden sogenannte Tarifbereichstypen ermittelt, die eine Charakterisierung von Branchentarifverträgen zulassen. Auf Basis dieser Analyse lassen sich die im IAW-Öffnungsklauseldatensatz gewonnenen Informationen mit anderen Datenquellen, z.B. dem IAB-Betriebspanel, verknüpfen.

Spindler (2007) untersucht die Einführung tarifvertraglicher Öffnungsklauseln in den 1990er Jahren unter Berücksichtigung von Branchen- und Betriebscharakteristika und der jeweiligen wirtschaftlichen Lage mit Hilfe eines PanelAnsatzes. Aus den Ergebnissen lässt sich - als Grundintention einer Flexibilisierung durch tarifvertragliche Öffnungsklauseln - die Reaktion auf betriebliche Schocks (steigende Arbeitskosten) bei gleichzeitigem Festhalten an übergeordneten tariflichen Strukturen (abnehmender Anteil tarifgebundener Betriebe) erkennen.

Heinbach und Schröpfer (2008) knüpfen daran an, indem sie die Bedingungen untersuchen, die zu einer tatsächlichen betrieblichen Anwendung tariflicher Öffnungsklauseln führen. Auf Basis des IAB-Betriebspanels testen sie Hypothesen aus neueren Handelstheorien zu Produktivitätsunterschieden zwischen Firmen einer Branche. Die Exportorientierung eines Betriebs als Maß für dessen Produktivität ist mit einer bestimmten Arbeitsnachfrage verbunden. Damit ergeben sich bei Betrieben unterschiedlicher Exportorientierung und Produktivität auch Unterschiede in der Wahrscheinlichkeit, Tariföffnungsklauseln zu nutzen. Es 
wird deutlich, dass die in ihrer Intensität gestiegenen internationalen Handelsbeziehungen mit der Inzidenz von tariflicher Öffnung verbunden sind.

Inwiefern sich die Existenz sowie die Anwendung von Öffnungsklauseln auf die betriebliche Entwicklung auswirken, untersuchen Heinbach (2007) und Maier (2009) in Analysen über Höhe und Verteilung der Entlohnung. Der resultierende Gesamteffekt kann in theoretischer Hinsicht nicht eindeutig bestimmt werden. Heinbach (2007) nutzt die Verdienststrukturerhebung und unterscheidet erstmals zwischen Tarifregimen mit entgeltrelevanten oder arbeitszeitrelevanten Öffnungsklauseln im Vergleich zu kollektiv verhandelten Löhnen ohne Öffnungsklauseln. Im Ergebnis ist mit ersteren ein vergleichsweise geringes Lohnniveau verbunden, während bei arbeitszeitrelevanten Öffnungsklauseln höhere Löhne beobachtet werden können. Maier (2009) nutzt den Linked-Employer-Employee-Datensatz des IAB und untersucht auch die Anwendung von Öffnungsklauseln. Es zeigen sich gegenüber Flächentarifverträgen im Niveau geringere Löhne verbunden mit einer Kompression der Löhne, falls generell Öffnungsklauseln vorliegen. Keine wesentliche Veränderung dieses Ergebnisses ergibt sich unter zusätzlicher Berücksichtigung der Anwendung von Öffnungsklauseln. Tarifliche Öffnungsklauseln weisen daher ein gewisses Potenzial zur Flexibilisierung auf, sowohl was das Lohnniveau als auch die Lohnstruktur angeht. Aufgrund nicht beobachtbarer Selektionseffekte auf Seiten der Betriebe sind die Ergebnisse jedoch mit Vorsicht zu interpretieren, insbesondere was die Ableitung von wirtschaftspolitischen Implikationen im Hinblick auf die resultierende Lohnstruktur bei Existenz und Anwendung von Öffnungsklauseln betrifft.

Mit Blick auf die Beschäftigungsentwicklung untersuchen Brändle und Heinbach (2010) mit Daten des IABBetriebspanels das Beschäftigungswachstum von Betrieben. Wiederum sind aus theoretischer Sicht verschiedene Effekte möglich. Einerseits können Betriebe Öffnungsklauseln nutzen, um Beschäftigungsverluste zu vermeiden. Andererseits können Öffnungsklauseln dazu führen, dass Betriebe mehr Arbeitnehmer einstellen. Im Ergebnis zeigt sich, dass Betriebe mit Tariföffnungsklauseln ein größeres Beschäftigungswachstum aufweisen, was sich an einem geringeren Arbeitsplatzabbau zeigt. Eine konkrete Anwendung tariflicher Öffnungsklauseln ist jedoch nicht mit signifikant unterschiedlichen Beschäftigungsströmen verbunden.

Zusammenfassend hat das dargestellte Forschungsprojekt zum tieferen Verständnis des Ausmaßes sowie der Determinanten und der Wirkungen von tarifvertraglichen Flexibilisierungspotenzialen einen wesentlichen Beitrag geleistet. Demnach stellen Tariföffnungsklauseln ein zunehmend verbreitetes Instrument der Beschäftigungspolitik dar, welches innerhalb des bestehenden institutionellen Rahmens des deutschen Arbeitsmarktes Beschäftigung sichern kann. Dieses Instrument sollte daher erstens weiter ausgebaut und zweitens vermehrt publik gemacht werden.

\section{Executive summary}

Given the recent financial crisis, the German labour market performs relatively well. This has not been the case until recent years: collective bargaining and the rigid system of wage setting have been often cited as one of the reasons for Germany's high structural unemployment. Contrary, a reform process has started at least since the mid-1990s, introducing measures of flexibility and decentralization to the formally rigid system of wage setting.

This article summarises the developments of possible and actual flexibilisation of collective bargaining contracts via so called hardship or opening clauses. A literature review sums up the scientific progress that had been made at the beginning of the research project and shows which gaps were to fill. Lacking data, the first parts of the project investigate on the actual existence and shape of opening clauses and create a new data set to measure the incidence of opening clauses in collective bargaining agreements in West German manufacturing sector for the years 1995 to 2007, the IAW data set on opening clauses. Using this, we analyse the determinants of the introduction of opening clauses on the industry level and of their actual implementation on the firm level. The latter parts of the project focus on the relation between opening clauses and measures of firm performance, especially considering wages and job growth. The results show that opening clauses are more and more common and that a more diversified wage structure and higher job growth is observed.

\section{Literatur}

Abberger, K., Becker, S., Hofmann, B., Wohlrabe, K.: Mikrodaten im ifo Institut für Wirtschaftsforschung - Bestand, Verwendung und Zugang. AStA Wirtsch. Sozialstat. Arch. 1(1), 27-42 (2007)

Antoni, M., Jahn, E.J.: Do changes in regulation affect employment duration in temporary help agencies? Ind. Labor Relat. Rev. 62(2), 226-251 (2009)

Bernard, A., Eaton, B., Jensen, J., Kortum, J.: Plants and productivity in international trade. Am. Econ. Rev. 93(4), 1268-1290 (2003)

Bispinck, R.: Tarifliche Öffnungsklauseln. Eine Analyse von rund 30 Tarifbereichen. Elemente qualitativer Tarifpolitik Nr. 52, Düsseldorf (2003)

Brändle, T., Heinbach, W.D.: Opening clauses in collective bargaining agreements: more flexibility to save jobs? Diskussionspapier 67, IAW, Tübingen (2010)

Burda, M., Fitzenberger, B., Lembcke, A., Vogel, T.: Unionization, stochastic dominance, and compression of the wage distribution: evidence from Germany. Diskussionspapier SFB 649 08-41, Humboldt-Universität Berlin (2008)

Ellguth, P., Kohaut, S.: Tarifbindung und betriebliche Interessenvertretung - Aktuelle Ergebnisse aus dem IAB-Betriebspanel 2006. WSI-Mitt. 2007(9), 511-514 (2007)

Fitzenberger, B., Franz, W.: Flächentarifvertrag: Eine kritische Würdigung aus ökonomischer Sicht. In: Franz, W., Ramser, H.-J., Stadler, M. (Hrsg.). Ökonomische Analyse von Verträgen. Wirtschaftswissenschaftliches Seminar Ottobeuren, S. 191-232. Mohr Siebeck, Tübingen (1999) 
Fitzenberger, B., Kohn, K., Lembcke, A.: Union density and varieties of coverage: the anatomy of union wage effects in Germany. Diskussionspapier 08-012, ZEW, Mannheim (2008)

Franz, W., Pfeiffer, F.: Tarifbindung und die ökonomische Rationalität von Lohnrigiditäten. Jahrb. Natl.ökon. Stat. 233(1), 23-57 (2003)

Franz, W., Pfeiffer, F.: Reasons for wage rigidity in Germany. LABOUR 20(2), 255-284 (2006)

Gerlach, K., Stephan, G.: Bargaining regimes and wage dispersion. Jahrb. Natl.ökon. Stat. 226(6), 629-645 (2006)

Gürtzgen, N.: Das Forschungspotenzial von Linked EmployerEmployee Daten am Beispiel von Lohneffekten der Tarifbindung. AStA Wirtsch. Sozialstat. Arch. 2(2), 223-240 (2008)

Heinbach, W.D.: Ausmaß und Grad der tarifvertraglichen Öffnung. IAW Rep. 2005(2), 51-70 (2005)

Heinbach, W.D.: Immer mehr Tarifverträge sehen Öffnungsklauseln vor. Statist. Monatsheft Baden-Württ. 2006(11), 44-47 (2006)

Heinbach, W.D.: Wages in wage-setting regimes with opening clauses. AStA Wirtsch. Sozialstat. Arch. 1(3/4), 233-245 (2007)

Heinbach, W.D.: Tarifbindung, Lohnstruktur und tarifvertragliche Flexibilisierungspotenziale. IAW-Forschungsbericht Nr. 69, IAW, Tübingen (2009)

Heinbach, W.D., Schröpfer, S.: Typisierung der Tarifvertragslandschaft - Eine Clusteranalyse der tarifvertraglichen Öffnungsklauseln. Jahrb. Natl.ökon. Stat. 227(3), 219-235 (2007)

Heinbach, W.D., Schröpfer, S.: What a difference trade makes - export activity and the flexibility of collective bargaining agreements. Z. Arbeitsmarktforsch. 41(2/3), 287-303 (2008)

Hübler, O.: Sind betriebliche Bündnisse für Arbeit erfolgreich? Jahrb. Natl.ökon. Stat. 225(6), 630-652 (2005)

Kohaut, S., Schnabel, C.: Verbreitung, Ausmaß und Determinanten der übertariflichen Entlohnung. Mitt. Arb.markt- Berufsforsch. 4, 661-671 (2003a)

Kohaut, S., Schnabel, C.: Zur Erosion des Flächentarifvertrags: Ausmaß, Einflussfaktoren und Gegenmaßnahmen. Ind. Bezieh. 12(2), 193-219 (2003b)

Kohaut, S., Schnabel, C.: Tarifliche Öffnungsklauseln - Verbreitung, Inanspruchnahme und Bedeutung. Soz. Fortschr. 56(2), 33-40 (2007)

Kohn, K., Lembcke, A.: Wage distribution by bargaining regime Linked employer-employee data evidence from Germany. AStA Wirtsch. Sozialstat. Arch. 1(3/4), 247-261 (2007)

Kölling, A.: The IAB-establishment panel. Schmollers Jahrb. Wirtsch.Soz.wiss. 120(2), 291-300 (2000)

Maier, M.F.: Auswirkungen der Anwendung von tarifvertraglichen Öffnungsklauseln auf die Lohnstruktur. Eine empirische Analyse unter Verwendung der Linked-Employer-Employee-Daten des
IAB. Diplomarbeit, Fakultät Wirtschafts- und Sozialwissenschaften, Universität Hohenheim (2009)

OECD: OECD - STructural ANalysis (STAN) Database. Organisation for Economic Co-operation and Development, Paris (2008)

Spindler, M.: Determinanten der Einführung von tariflichen Öffnungsklauseln - Eine empirische Analyse mit Hilfe von Panel-Logit und Panel-Probit-Modellen. Diplomarbeit, Fakultät Wirtschafts- und Sozialwissenschaften, Universität Hohenheim (2007)

Tobias Brändle studierte Internationale Volkswirtschaftslehre, Englisch und Politik an der Eberhard-Karls-Universität Tübingen und der University of Maryland, College Park. Im März 2009 schloss er sein Studium als Diplom-Volkswirt mit der Arbeit „Tarifliche Lohnflexibilisierung und betriebliche Beschäftigungsentwicklung“ ab. Seit April 2009 arbeitet er als wissenschaftlicher Mitarbeiter an der Abteilung für Volkswirtschaftslehre, insbesondere Finanzwissenschaft der Wirtschafts- und Sozialwissenschaftlichen Fakultät der EberhardKarls-Universität Tübingen. Er forscht dort zum Thema „Labour Market Institutions and Firm Performance in Germany" bei Professor Dr. Laszlo Goerke.

Wolf Dieter Heinbach studierte Wirtschaftswissenschaften an der Universität Hohenheim und der Université des Sciences Sociales Toulouse, Frankreich. Er arbeitete seit Oktober 2003 am Institut für Angewandte Wirtschaftsforschung (IAW) Tübingen und am Lehrstuhl für Statistik und Ökonometrie an der Universität Hohenheim. Im Rahmen seiner Dissertation befasste er sich mit Tarifbindung, Lohnstruktur und tarifvertraglichen Flexibilisierungspotenzialen; Promotion im November 2008 bei Professor Dr. Gerhard Wagenhals (Universität Hohenheim) und Professor Dr. Gerd Ronning (Universität Tübingen). Seit Januar 2009 ist er beim Ministerium für Wissenschaft, Forschung und Kunst Baden-Württemberg im Bereich Fortentwicklung des Hochschulwesens und Hochschulstatistik beschäftigt.

Michael F. Maier studierte Wirtschaftswissenschaften an der Universität Hohenheim mit den Schwerpunkten Ökonometrie, Arbeitsmarktökonomik und Finanzwissenschaften. Im Juli 2009 schloss er sein Studium als Diplom-Ökonom ab. In seiner Diplomarbeit untersuchte er die Auswirkungen von tarifvertraglichen Öffnungsklauseln auf die Lohnstruktur. Seit September 2009 ist Michael Maier als Wissenschaftler am Zentrum für Europäische Wirtschaftsforschung (ZEW) im Forschungsbereich „Arbeitsmärkte, Personalmanagement und Soziale Sicherung" beschäftigt. Sein Forschungsschwerpunkt ist die Evaluation aktiver Arbeitsmarktpolitik. 